\title{
Stable Analysis of Solution Set for System of Quasivariational Relations with Applications
}

\author{
Lefeng Shi ${ }^{1,2}$ and Zhe Yang ${ }^{3,4}$ \\ ${ }^{1}$ State Grid Chongqing Electric Power Research Institute, State Grid Chongqing Electric Power Corporation, Chongqing 401123, China \\ ${ }^{2}$ College of Economics and Business Administration, Chongqing University, Chongqing 400044, China \\ ${ }^{3}$ School of Economics, Shanghai University of Finance and Economics, Shanghai 200433, China \\ ${ }^{4}$ Key Laboratory of Mathematical Economics (SUFE), Ministry of Education, Shanghai 200433, China
}

Correspondence should be addressed to Zhe Yang; zheyang211@163.com

Received 9 April 2014; Revised 22 July 2014; Accepted 22 July 2014; Published 12 August 2014

Academic Editor: Naseer Shahzad

Copyright (c) 2014 L. Shi and Z. Yang. This is an open access article distributed under the Creative Commons Attribution License, which permits unrestricted use, distribution, and reproduction in any medium, provided the original work is properly cited.

\begin{abstract}
The essential stability of solutions for system of quasivariational relations is studied. We show that most of systems of quasivariational relations are essential (in the sense of Baire category) and that, for any system of quasivariational relations, there exists at least one essential component of its solution set. As applications, the existence of essential components of solution set for systems of KKM problems and systems of quasivariational inclusions is obtained.
\end{abstract}

\section{Introduction}

It is well known that the equilibrium problem is a unified model of several problems, namely, optimization problem, saddle point problem, variational inequality problem, fixed point problem, Nash equilibrium problem, and so forth. Recently, several people focused on the study of equilibrium problems and their generalizations. Luc [1] introduced a more general model of equilibrium problems which is called a variational relation problem (in short, VR). The stability of the solution set of variational relation problems was studied in $[2,3]$. Various types of sufficient conditions for the existence of solutions of variational relation problems have been investigated in many recent papers (see [4-12]). Furthermore, Agarwal et al. [13] presented a unified approach for studying the existence of solutions for two types of variational relation problems, and Balaj and Lin [14] established the existence criteria for the solutions of two very general types of variational relation problems.

However, One could also argue that a sensible equilibrium should be stable against slight perturbations in the payoffs of the game (van Damme [15]). The notation of an essential solution for fixed points was firstly introduced by Fort [16], which means that, for a fixed point $x$ of a mapping $f$, if each mapping sufficiently near to $f$ has a fixed point arbitrarily near to $x, x$ is said to be essential. The method of essential solution has been widely used in various fields recently. It plays a crucial role in the study of stability of solutions including optimal solutions, Nash equilibria, and fixed points (see [17-30]).

Motivated and inspired by research works mentioned above, in this paper, we study the notions of essential stability of solutions for system of quasivariational relations. The results of this paper improve and generalize several known results on the stability of solution set for variational relation problems.

\section{Definitions and Preliminaries}

Lin and Ansari [8] introduced a system of quasivariational relations (SQVP) and established the existence of solutions of SQVP by means of maximal element theorem for a family of multivalued mappings. Let $I$ be any index set. For each $i \in I$, let $X_{i}$ be nonempty set, let $X=\prod_{i \in I} X_{i}, S_{i}, Q_{i}: X \rightrightarrows$ $X_{i}$ be multivalued mappings with nonempty values, and let $R_{i}\left(x, y_{i}\right)$ be a relation linking $x \in X$ and $y_{i} \in X_{i}$. A system $q=\left(I, X_{i}, Q_{i}, S_{i}, R_{i}\right)$ of quasivariational relations consists in finding $x^{*} \in X$ such that, for each $i \in I, x_{i}^{*} \in S_{i}\left(x^{*}\right)$ and $R_{i}\left(x^{*}, y_{i}\right)$ holds for any $y_{i} \in Q_{i}\left(x^{*}\right)$. 
Definition 1. Let $I$ be a finite set, and $z^{0} \in X$. Let $\mathscr{M}$ be the set of all systems of quasivariational relations $q=\left(I, X_{i}\right.$, $Q_{i}, S_{i}, R_{i}$ ) such that the following hold: (i) for each $i \in I, X_{i}$ is nonempty convex compact subset of a normed linear space; (ii) for each $i \in I, S_{i}$ is upper semicontinuous with nonempty convex compact values; (iii) for each $i \in I, Q_{i}^{-1}\left(y_{i}\right)$ is open in $X$ for any $y_{i} \in X_{i}$; (iv) $z_{i}^{0} \in Q_{i}(x) \subset S_{i}(x)$ for any $x \in X$ and any $i \in I$; (v) for each $i \in I$, any finite set $\left\{x_{i 1}, \ldots, x_{i n}\right\} \subset X_{i}$, and any $x_{i} \in \operatorname{co}\left\{x_{i 1}, \ldots, x_{i n}\right\}$, there is $j \in\{1, \ldots, n\}$ such that $R_{i}\left(x, x_{i j}\right)$ holds, where $x=\left(x_{i}\right)_{i \in I}$; (vi) $R_{i}\left(\cdot, y_{i}\right)$ is closed for any $y_{i} \in X_{i}$.

Clearly, for any $q \in \mathscr{M}$, we have that (1) $\left\{x \in X: x_{i} \in\right.$ $\left.S_{i}(x)\right\}$ is closed; (2) $Q_{i}(x) \neq \emptyset$ for all $x \in X$; (3) since $S_{i}(x)$ is convex and $Q_{i}(x) \subset S_{i}(x)$, then $\operatorname{co} Q_{i}(x) \subset S_{i}(x)$. By Theorem 3.1 of [8], the problem $q$ has at least one solution. Here, for any $q \in \mathscr{M}$ and each $i \in I$, define two closed-valued mappings $A_{i}, B_{i}: X_{i} \rightrightarrows X$ by $A_{i}\left(y_{i}\right)=\left\{x \in X: y_{i} \notin Q_{i}(x)\right\}$ and $B_{i}\left(y_{i}\right)=\left\{x \in X: R_{i}\left(x, y_{i}\right)\right.$ holds $\}$. Denote by $F(q)$ the solution set of $q$. Thus, a correspondence $F: \mathscr{M} \rightrightarrows X$ is well defined. For each $q, q^{\prime} \in \mathscr{M}$, define the distance on $\mathscr{M}$ by

$$
\begin{aligned}
\rho\left(q, q^{\prime}\right)= & \sup _{i \in I} \sup _{x \in X} h_{i}\left(S_{i}(x), S_{i}^{\prime}(x)\right) \\
& +\sup _{i \in I} \sup _{y_{i} \in X_{i}} h\left(A_{i}\left(y_{i}\right), A_{i}^{\prime}\left(y_{i}\right)\right) \\
& +\sup _{i \in I} \sup _{y_{i} \in X_{i}} h\left(B_{i}\left(y_{i}\right), B_{i}^{\prime}\left(y_{i}\right)\right),
\end{aligned}
$$

where $h_{i}$ is the Hausdorff distance on $X_{i}$ and $h$ is the Hausdorff distance on $X$. Clearly, $(\mathscr{M}, \rho)$ is a metric space.

In what follows, the notions of essential solutions, essential problems are introduced (see [25]). Let $(X, d),(Y, \rho)$ be two metric spaces, and let $h$ be the Hausdorff distance defined on $Y$. A set-valued mapping $F: X \rightrightarrows Y$ is said to be (1) upper semicontinuous at $x \in X$ if, for any open subset $O$ of $Y$ with $O \supset F(x)$, there exists an open neighborhood $U(x)$ of $x$ such that $O \supset F\left(x^{\prime}\right)$ for any $x^{\prime} \in U(x)$; (2) upper semicontinuous on $X$ if $F$ is upper semicontinuous on each $x \in X$; (3) an usco mapping if $F$ is upper semicontinuous on $X$ and $F(x)$ is compact for each $x \in X$; (4) lower semicontinuous at $x \in X$ if, for any open subset $O$ of $Y$ with $O \cap F(x) \neq \emptyset$, there exists an open neighborhood $U(x)$ of $x$ such that $O \cap F\left(x^{\prime}\right) \neq \emptyset$ for any $x^{\prime} \in U(x)$; (5) lower semicontinuous on $X$ if $F$ is lower semicontinuous on each $x \in X$; (6) closed if $\operatorname{Graph}(F)=$ $\{(x, y) \in X \times Y \mid y \in F(x)\}$ is closed. Let $x \in X$. A point $y \in F(x)$ is said to be an essential point of $F(x)$ if, for any open neighborhood $N(y)$ of $y$ in $Y$, there is a $\delta>0$ such that $N(y) \cap F\left(x^{\prime}\right) \neq \emptyset$ for any $x^{\prime} \in X$ with $d\left(x, x^{\prime}\right)<\delta$. If all $y \in F(x)$ are essential, then $x$ is said to be essential. A nonempty closed subset $e(x)$ of $F(x)$ is said to be an essential set of $F(x)$ if, for any open set $U, e(x) \subset U$, there is a $\delta>0$ such that $U \cap F\left(x^{\prime}\right) \neq \emptyset$ for any $x^{\prime} \in X$ with $d\left(x, x^{\prime}\right)<\delta$. An essential subset $m(x) \subset F(x)$ is said to be a minimal essential set of $F(x)$ if it is a minimal element of the family of essential sets ordered by set inclusion. A component $C(x)$ is called an essential component of $F(x)$ if $C(x)$ is essential.
Remark 2 (see [25]). (1) It is easy to see that the problem $x \in$ $X$ is essential if and only if the mapping $F: X \rightrightarrows Y$ is lower semicontinuous at $x$. (2) Let two nonempty closed sets be $e_{1}$, $e_{2}$ of $F(x)$, if $e_{1} \subset e_{2}$, and $e_{1}$ is essential, so is $e_{2}$.

Lemma 3 (see [31]). If $X$ is a complete metric space and $F$ : $X \rightrightarrows Y$ is usco, then the set of points, where $F$ is lower semicontinuous, is a dense residual set in $X$.

Lemma 4 (see [22]). Let $C, D$ be two nonempty, convex, and compact subsets of linear normed space $Y$. Then $h(C, \lambda C+$ $\mu D) \leq h(C, D)$, where $\lambda, \mu \geq 0, \lambda+\mu=1$.

Lemma 5 (see $[24])$. Let $(Y, \rho)$ be a metric space, let $K_{1}$ and $K_{2}$ be two nonempty compact subsets of $Y$, and let $V_{1}$ and $V_{2}$ be two nonempty disjoint open subsets of $Y$. If $h\left(K_{1}, K_{2}\right)<\rho\left(V_{1}, V_{2}\right)$, then

$$
h\left(K_{1},\left(K_{1} \backslash V_{2}\right) \cup\left(K_{2} \backslash V_{1}\right)\right) \leq h\left(K_{1}, K_{2}\right) .
$$

Lemma 6 (see [32]). Let $X$ and $Y$ be two Hausdorff topological spaces with $Y$ compact. If $F$ is a closed set-valued mapping from $X$ to $Y$, then $F$ is upper semicontinuous.

Lemma 7 (see [23]). Let $X, Y, Z$ be three metric spaces, and let $S_{1}: Y \rightrightarrows X$ and $S_{2}: Z \rightrightarrows X$ be two set-valued mappings. Suppose that there exists at least one essential component of $S_{1}(y)$ for each $y \in Y$ and there exists a continuous singlevalued mapping $T: Z \rightarrow Y$ such that $S_{2}(z) \supset S_{1}(T(z))$ for each $z \in Z$. Then, there exists at least one essential component of $S_{2}(z)$ for each $z \in Z$.

\section{Main Results}

Theorem 8. $(\mathscr{M}, \rho)$ is a complete metric space.

Proof. Let $\left\{q^{n}\right\}_{n=1}^{\infty}$ be any Cauchy sequence in $\mathscr{M}$, and then, for any $\varepsilon>0$, there is $N>0$ such that $\rho\left(q^{n}, q^{m}\right)<\varepsilon$ for any $n, m>N$; that is,

$$
\begin{gathered}
\sup _{i \in I} \sup _{x \in X} h_{i}\left(S_{i}(x), S_{i}^{\prime}(x)\right)+\sup _{i \in I} \sup _{y_{i} \in X_{i}} h\left(A_{i}\left(y_{i}\right), A_{i}^{\prime}\left(y_{i}\right)\right) \\
+\sup _{i \in I} \sup _{y_{i} \in X_{i}} h\left(B_{i}\left(y_{i}\right), B_{i}^{\prime}\left(y_{i}\right)\right) \leq \varepsilon,
\end{gathered}
$$

for any $n, m>N$.

(1) Easily, for each $i \in I$, there exists a set-valued mapping $S_{i}: X \rightrightarrows X_{i}$ such that $S_{i}^{n}(x) \rightarrow S_{i}(x)$ for any $x \in X$ and $S_{i}$ is upper semicontinuous with nonempty compact convex values.

(2) For each $i \in I$, there exist two closed-valued mappings $A_{i}, B_{i}: X_{i} \rightrightarrows X$ such that, for any $y_{i} \in X_{i}$,

$$
A_{i}^{n}\left(y_{i}\right) \longrightarrow A_{i}\left(y_{i}\right), \quad B_{i}^{n}\left(y_{i}\right) \longrightarrow B_{i}\left(y_{i}\right) .
$$

Further, for each $i \in I$, define the mapping $Q_{i}: X \rightrightarrows$ $X_{i}$ and relation $R_{i}$ by the following:

$$
\begin{gathered}
Q_{i}(x)=\left\{y_{i} \in X_{i} \mid x \notin A_{i}\left(y_{i}\right)\right\}, \\
R_{i}\left(x, y_{i}\right) \text { holds if and only if } x \in B_{i}\left(y_{i}\right) .
\end{gathered}
$$

We need to prove that $q=\left(S_{i}, Q_{i}, R_{i}\right)_{i \in I} \in \mathscr{M}$. 
(3) Easily, for each $i \in I$ and any $y_{i} \in X_{i}, Q_{i}^{-1}\left(y_{i}\right)=$ $X \backslash A_{i}\left(y_{i}\right)$ is open in $X$. And, since $B_{i}\left(y_{i}\right)$ is closed, it follows that $R_{i}\left(\cdot, y_{i}\right)$ is closed.

(4) If there exist $i_{0} \in I$ and $x \in X$ such that $z_{i_{0}}^{0} \notin$ $Q_{i_{0}}(x)$, then $x \in A_{i_{0}}\left(z_{i_{0}}^{0}\right)$. It follows from $A_{i_{0}}^{n}\left(z_{i_{0}}^{0}\right) \rightarrow$ $A_{i_{0}}\left(z_{i_{0}}^{0}\right)$ that there exists a sequence $\left\{x^{n}\right\}$ of $X$ such that $x^{n} \in A_{i_{0}}^{n}\left(z_{i_{0}}^{0}\right)$ and $x^{n} \rightarrow x$, which implies that $z_{i_{0}}^{0} \notin$ $Q_{i_{0}}^{n}\left(x^{n}\right)$. It is a contradiction. Thus $z_{i}^{0} \in Q_{i}(x)$ for any $x \in X$ and any $i \in I$.

(5) Suppose the existence of $i \in I$ and $x \in X$ such that $Q_{i}(x) \not \subset S_{i}(x)$, and then there exist $y_{i} \in Q_{i}(x)$ such that $y_{i} \notin S_{i}(x)$. From $y_{i} \in Q_{i}(x)$, that is, $x \notin A_{i}\left(y_{i}\right)$, it follows that $x \notin A_{i}^{n}\left(y_{i}\right)$ for enough large $n$; that is, $y_{i} \in$ $Q_{i}^{n}(x)$. Moreover, since $y_{i} \notin S_{i}(x)$, then $y_{i} \notin S_{i}^{n}(x)$ for enough large $n$. Thus, for enough large $n, y_{i} \in Q_{i}^{n}(x)$ and $y_{i} \notin S_{i}^{n}(x)$, which implies that $Q_{i}^{n}(x) \not \subset S_{i}^{n}(x)$. It is a contradiction. Thus $Q_{i}(x) \subset S_{i}(x)$ for any $i \in I$ and any $x \in X$.

(6) Suppose that there exist $i_{0} \in I$, finite set $\left\{x_{i 1}, \ldots, x_{i m}\right\}$, for all $i \in I$, and $x=\left(x_{i}\right)_{i \in I} \in X$ with $x_{i} \in$ $\operatorname{co}\left\{x_{i 1}, \ldots, x_{i m}\right\}$, for all $i \in I$ such that $R_{i_{0}}\left(x, x_{i_{0} j}\right)$ does not hold for any $j \in\{1, \ldots, m\}$, and then $x \notin B_{i_{0}}\left(x_{i_{0} j}\right)$ for any $j \in\{1, \ldots, m\}$. Since $B_{i}^{n}\left(y_{i}\right) \rightarrow B_{i}\left(y_{i}\right)$ for any $y_{i} \in X_{i}$ and any $i \in I$, then, for enough large $n$, $x \notin B_{i_{0}}^{n}\left(x_{i_{0} j}\right)$ for any $j \in\{1, \ldots, m\}$, which implies that $R_{i_{0}}^{n}\left(x, x_{i_{0} j}\right)$ does not hold for any $j \in\{1, \ldots, m\}$. It is a contradiction. Thus, for each $i \in I$, any finite set $\left\{x_{i 1}, \ldots, x_{i n}\right\} \subset X_{i}$, and any $x_{i} \in \operatorname{co}\left\{x_{i 1}, \ldots, x_{i n}\right\}$, there is $j \in\{1, \ldots, n\}$ such that $R_{i}\left(x, x_{i j}\right)$ holds, where $x=\left(x_{i}\right)_{i \in I}$. Hence $q=\left(S_{i}, Q_{i}, R_{i}\right)_{i \in I} \in \mathscr{M}$. This completes the proof.

Theorem 9. The mapping $F: \mathscr{M} \rightrightarrows X$ is upper semicontinuous with compact values.

Proof. By Lemma 6, we only prove that $\operatorname{Graph}(F)$ is closed. For any $\left\{q^{n} \in \mathscr{M}\right\}_{n=1}^{\infty}$ with $q^{n} \rightarrow q$ and any $x^{n} \in F\left(q^{n}\right)$ with $x^{n} \rightarrow x$, we will prove $x \in F(q)$.

It follows from $x^{n} \in F\left(q^{n}\right)$ for any $n$ that, for each $i \in I$, $x_{i}^{n} \in S_{i}^{n}\left(x^{n}\right)$ and $R_{i}^{n}\left(x^{n}, y_{i}\right)$ holds for any $y_{i} \in Q_{i}\left(x^{n}\right)$. Since $q^{n} \rightarrow q$ and $x^{n} \rightarrow x$, then $x_{i} \in S_{i}(x)$ for any $i \in I$. Next, if there exist $i_{0} \in I$ and $y_{i_{0}} \in Q_{i_{0}}(x)$ such that $R_{i_{0}}\left(x, y_{i_{0}}\right)$ does not hold, then $x \notin A_{i_{0}}\left(y_{i_{0}}\right)$ and $x \notin B_{i_{0}}\left(y_{i_{0}}\right)$. Then, for enough large $n, x^{n} \notin A_{i_{0}}^{n}\left(y_{i_{0}}\right)$ and $x^{n} \notin B_{i_{0}}^{n}\left(y_{i_{0}}\right)$, which implies that $y_{i_{0}} \in Q_{i_{0}}^{n}\left(x^{n}\right)$ and $R_{i_{0}}^{n}\left(x^{n}, y_{i_{0}}\right)$ does not hold for enough large $n$. It is a contradiction. Thus, for each $i \in I, x_{i} \in S_{i}(x)$ and $R_{i}\left(x, y_{i}\right)$ holds for any $y_{i} \in Q_{i}(x)$; that is, $x \in F(q)$. This completes the proof.

Theorem 10. There exists a dense residual subset $\mathscr{G}$ of $\mathscr{M}$ such that, for each $q \in \mathscr{G}, q$ is essential.

Proof. Since $(\mathscr{M}, \rho)$ is complete by Theorem 8 , and the mapping $F: \mathscr{M} \rightrightarrows X$ is upper semicontinuous with nonempty compact values by Theorem 9, by Lemma 3, there is a dense residual subset $\mathscr{G}$ of $\mathscr{M}$, where $F$ is lower semicontinuous; thus, $q$ is essential for each $q \in \mathscr{G}$ by Remark $2(1)$.

Theorem 11. For each $q \in \mathscr{M}$, there exists at least one minimal essential subset of $F(q)$.

Proof. By Theorem 9, $F: \mathscr{M} \rightrightarrows X$ is upper semicontinuous with nonempty compact values; that is, for each open set $O \supset F(q)$, there exists $\delta>0$ such that, for any $q^{\prime} \in \mathscr{M}$ with $\rho\left(q, q^{\prime}\right)<\delta, O \supset F\left(q^{\prime}\right)$. Hence $F(q)$ is an essential set of itself. Let $\Theta$ denote the family of all essential sets of $F(q)$ ordered by set inclusion. Then $\Theta$ is nonempty and every decreasing chain of elements in $\Theta$ has a lower bound (because by the compactness the intersection is in $\Theta$ ); therefore, by Zorn's lemma, $\Theta$ has a minimal element and this minimal element is a minimal essential set of $F(q)$.

Theorem 12. For each $q \in M$, there exists at least one connected minimal essential subset of $F(q)$.

Proof. For each $q \in \mathscr{M}$, let $m(q) \subset F(q)$ be a minimal essential subset of $F(q)$. Suppose that $m(q)$ was not connected, then there exist two nonempty compact subsets $c_{1}(q), c_{2}(q)$ with $m(q)=c_{1}(q) \cup c_{2}(q)$, and there exist two disjoint open subsets $V^{1}, V^{2}$ in $X$ such that $V^{1} \supset c_{1}(q)$ and $V^{2} \supset c_{2}(q)$. Since $m(q)$ is a minimal essential set of $F(q)$, neither $c_{1}(q)$ nor $c_{2}(q)$ is essential. There exist two open sets $O^{1} \supset c_{1}(q)$ and $O^{2} \supset c_{2}(q)$ such that, for any $\delta>0$, there exist $q^{1}, q^{2} \in \mathscr{M}$ with

$$
\begin{aligned}
\rho\left(q, q^{1}\right)<\delta, & \rho\left(q, q^{2}\right)<\delta, \\
F\left(q^{1}\right) \cap O^{1}=\emptyset, & F\left(q^{2}\right) \cap O^{2}=\emptyset .
\end{aligned}
$$

Now, we choose two open sets $W^{1}, W^{2}$ such that

$$
\begin{aligned}
& c_{1}(q) \subset W^{1} \subset \bar{W}^{1} \subset O^{1} \cap V^{1}, \\
& c_{2}(q) \subset W^{2} \subset \bar{W}^{2} \subset O^{2} \cap V^{2},
\end{aligned}
$$

and $\inf \left\{d(a, b) \mid a \in W^{1}, b \in W^{2}\right\}=\varepsilon>0$.

Since $m(q)$ is essential, then, for $m(q) \subset\left(W^{1} \cup W^{2}\right)$, there exists $0<\delta^{*}<\varepsilon$ such that $F\left(q^{\prime}\right) \cap\left(W^{1} \cup W^{2}\right) \neq \emptyset$ for any $q^{\prime} \in \mathscr{M}$ with $\rho\left(q, q^{\prime}\right)<\delta^{*}$. Since $m(q)$ is a minimal essential set of $F(q)$, then neither $c_{1}(q)$ nor $c_{2}(q)$ is essential. Thus, for $\delta^{*} / 16>0$, there exist two $q^{1}, q^{2} \in \mathscr{M}$ such that

$$
\begin{array}{cl}
F\left(q^{1}\right) \cap W^{1}=\emptyset, & F\left(q^{2}\right) \cap W^{2}=\emptyset, \\
\rho\left(q^{1}, q\right)<\frac{\delta^{*}}{16}, & \rho\left(q^{2}, q\right)<\frac{\delta^{*}}{16} .
\end{array}
$$

Thus $\rho\left(q^{1}, q^{2}\right)<\delta^{*} / 8$. 
Now, define the following system of quasivariational relations $q^{\prime}=\left(S_{i}^{\prime}, Q_{i}^{\prime}, R_{i}^{\prime}\right)_{i \in I}$ by

$$
\begin{gathered}
S_{i}^{\prime}(x)=\lambda(x) S_{i}^{1}(x)+\mu(x) S_{i}^{2}(x), \quad \forall x \in X, \quad \forall i \in I, \\
A_{i}^{\prime}\left(y_{i}\right)=\left(A_{i}^{1}\left(y_{i}\right) \backslash W^{2}\right) \cup\left(A_{i}^{2}\left(y_{i}\right) \backslash W^{1}\right), \\
\forall y_{i} \in X_{i}, \quad \forall i \in I, \\
B_{i}^{\prime}\left(y_{i}\right)=\left(B_{i}^{1}\left(y_{i}\right) \backslash W^{2}\right) \cup\left(B_{i}^{2}\left(y_{i}\right) \backslash W^{1}\right), \\
\forall y_{i} \in X_{i}, \quad \forall i \in I, \\
Q_{i}^{\prime}(x)=\left\{y_{i} \in X_{i}: x \notin A_{i}\left(y_{i}\right)\right\}, \quad \forall x \in X, \quad \forall i \in I, \\
R_{i}^{\prime}\left(x, y_{i}\right) \text { holds if and only if } x \in B_{i}^{\prime}\left(y_{i}\right), \quad \forall i \in I,
\end{gathered}
$$

where

$$
\begin{gathered}
\lambda(x)=\frac{d\left(x, \bar{W}^{2}\right)}{d\left(x, \bar{W}^{1}\right)+d\left(x, \bar{W}^{2}\right),} \\
\mu(x)=\frac{d\left(x, \bar{W}^{1}\right)}{d\left(x, \bar{W}^{1}\right)+d\left(x, \bar{W}^{2}\right)}, \\
\forall x \in X, \\
A_{i}^{1}\left(y_{i}\right)=\left\{x \in X: y_{i} \notin Q_{i}^{1}(x)\right\}, \\
A_{i}^{2}\left(y_{i}\right)=\left\{x \in X: y_{i} \notin Q_{i}^{2}(x)\right\}, \\
B_{i}^{1}\left(y_{i}\right)=\left\{x \in X: R_{i}^{1}\left(x, y_{i}\right) \text { holds }\right\}, \\
B_{i}^{2}\left(y_{i}\right)=\left\{x \in X: R_{i}^{2}\left(x, y_{i}\right) \text { holds }\right\} .
\end{gathered}
$$

Easily, we can check the following.

(i) $S_{i}^{\prime}$ is upper semicontinuous with nonempty compact convex values for each $i \in I$.

(ii) $Q_{i}^{\prime-1}\left(y_{i}\right)=X \backslash A_{i}^{1}\left(y_{i}\right)$ is open for any $y_{i} \in X_{i}$ and any $i \in I$.

(iii) For any $i \in I$ and any $y_{i} \in X_{i}$, since $B_{i}^{1}\left(y_{i}\right)$ is closed, then $R_{i}\left(\cdot, y_{i}\right)$ is closed.

(iv) For each $i \in I$, since $z_{i}^{0} \in Q_{i}^{1}(x)$ and $z_{i}^{0} \in Q_{i}^{2}(x)$ for any $x \in X$, then $x \notin A_{i}^{1}\left(z_{i}^{0}\right)$ and $x \notin A_{i}^{2}\left(z_{i}^{0}\right)$ for any $x \in X$, which implies that $x \notin A_{i}^{1}\left(z_{i}^{0}\right)$ for any $x \in X$; that is, $z_{i}^{0} \in Q_{i}^{\prime}(x)$ for any $x \in X$.

(v) Next, we show $Q_{i}^{\prime}(x) \subset S_{i}^{\prime}(x)$ for any $x \in X$ and any $i \in I$. For any fixed $i \in I$, we have three cases.

If $x \in W^{1}$, then $Q_{i}^{\prime}(x)=Q_{i}^{1}(x)$ and $S_{i}^{\prime}(x)=$ $S_{i}^{1}(x)$. It follows from $q^{1} \in \mathscr{M}$ that

$$
Q_{i}^{\prime}(x)=Q_{i}^{1}(x) \subset S_{i}^{1}(x)=S_{i}^{\prime}(x) .
$$

If $x \in W^{2}$, then $Q_{i}^{\prime}(x)=Q_{i}^{2}(x)$ and $S_{i}^{\prime}(x)=$ $S_{i}^{2}(x)$. It follows from $q^{2} \in \mathscr{M}$ that

$$
Q_{i}^{\prime}(x)=Q_{i}^{2}(x) \subset S_{i}^{2}(x)=S_{i}^{\prime}(x) .
$$

If $x \in X \backslash\left(W^{1} \cup W^{2}\right)$, then $Q_{i}^{\prime}(x)=Q_{i}^{1}(x) \cap Q_{i}^{2}(x)$ and $S_{i}^{\prime}(x)=\lambda(x) S_{i}^{1}(x)+\mu(x) S_{i}^{2}(x)$. For any $z_{i} \epsilon$ $Q_{i}^{\prime}(x)=Q_{i}^{1}(x) \cap Q_{i}^{2}(x)$, we have $z_{i} \in Q_{i}^{1}(x) \subset$ $S_{i}^{1}(x)$ and $z_{i} \in Q_{i}^{2}(x) \subset S_{i}^{2}(x)$. Then

$$
z_{i}=\lambda(x) z_{i}+\mu(x) z_{i} \in \lambda(x) S_{i}^{1}(x)+\mu(x) S_{i}^{2}(x)=S_{i}^{\prime}(x) .
$$

Thus $Q_{i}^{\prime}(x) \subset S_{i}^{\prime}(x)$.

(vi) Suppose that there exist $i_{0} \in I$, finite set $\left\{x_{i 1}, \ldots, x_{i m}\right\}$, for all $i \in I$, and $x=\left(x_{i}\right)_{i \in I} \in X$ with $x_{i} \in \operatorname{co}\left\{x_{i 1}\right.$, $\left.\ldots, x_{i m}\right\}$, for all $i \in I$ such that $R_{i_{0}}^{\prime}\left(x, x_{i_{0} j}\right)$ does not hold for any $j \in\{1, \ldots, m\}$, and then $x \notin B_{i_{0}}^{\prime}\left(x_{i_{0} j}\right)$ for any $j \in\{1, \ldots, m\}$. Since $W^{1} \cap W^{2}=\emptyset$, without loss of generality, we assume $x \notin W^{1}$. For any $j \in\{1, \ldots, m\}$, since $x \notin B_{i_{0}}^{\prime}\left(x_{i_{0} j}\right)=\left(B_{i_{0}}^{1}\left(x_{i_{0} j}\right) \backslash W^{2}\right) \cup\left(B_{i_{0}}^{2}\left(x_{i_{0} j}\right) \backslash W^{1}\right)$, then $x \notin B_{i_{0}}^{2}\left(x_{i_{0} j}\right) \backslash W^{1}$, for all $j \in\{1, \ldots, m\}$. It follows from $x \notin W^{1}$ that $x \notin B_{i_{0}}^{2}\left(x_{i_{0} j}\right), \forall j \in\{1, \ldots, m\}$; that is, $R_{i_{0}}^{2}\left(x, x_{i_{0} j}\right)$ does not hold for any $j \in\{1, \ldots, m\}$. It is a contradiction.

(vii) By Lemmas 4 and 5, we have

$$
\begin{aligned}
\rho\left(q^{\prime}, q\right)= & \sup _{i \in I} \sup _{x \in X} h_{i}\left(S_{i}(x), S_{i}^{\prime}(x)\right) \\
& +\sup _{i \in I} \sup _{y_{i} \in X_{i}} h\left(A_{i}\left(y_{i}\right), A_{i}^{\prime}\left(y_{i}\right)\right) \\
& +\sup _{i \in I} \sup _{y_{i} \in X_{i}} h\left(B_{i}\left(y_{i}\right), B_{i}^{\prime}\left(y_{i}\right)\right) \\
\leq & \sup _{i \in I} \sup _{x \in X} h_{i}\left(S_{i}(x), S_{i}^{1}(x)\right) \\
& +\sup _{i \in I} \sup _{x \in X} h_{i}\left(S_{i}^{1}(x), S_{i}^{\prime}(x)\right) \\
& +\sup _{i \in I} \sup _{y_{i} \in X_{i}} h\left(A_{i}\left(y_{i}\right), A_{i}^{1}\left(y_{i}\right)\right) \\
& +\sup _{i \in I} \sup _{y_{i} \in X_{i}} h\left(A_{i}^{1}\left(y_{i}\right), A_{i}^{\prime}\left(y_{i}\right)\right) \\
& +\sup _{i \in I} \sup _{y_{i} \in X_{i}} h\left(B_{i}\left(y_{i}\right), B_{i}^{1}\left(y_{i}\right)\right) \\
& +\sup _{i \in I} \sup _{y_{i} \in X_{i}} h\left(A_{i}^{1}\left(y_{i}\right), A_{i}^{2}\left(y_{i}\right)\right) \\
& +\sup _{i \in I} \sup _{y_{i} \in X_{i}} h\left(B_{i}^{1}\left(y_{i}\right), B_{i}^{\prime}\left(y_{i}\right)\right) \\
\leq & \sup _{i \in I} \sup _{x \in X} h_{i}\left(S_{i}(x), S_{i}^{1}(x)\right) \\
& +\sup _{i \in I} \sup _{x \in X} h_{i}\left(S_{i}^{1}(x), S_{i}^{2}(x)\right) \\
& \\
& \\
&
\end{aligned}
$$




$$
\begin{aligned}
& \quad+\sup _{i \in I} \sup _{y_{i} \in X_{i}} h\left(B_{i}\left(y_{i}\right), B_{i}^{1}\left(y_{i}\right)\right) \\
& +\sup _{i \in I} \sup _{y_{i} \in X_{i}} h\left(B_{i}^{1}\left(y_{i}\right), B_{i}^{2}\left(y_{i}\right)\right) \\
& \leq 3\left(\frac{1}{16}+\frac{2}{16}\right) \delta^{*} \\
& <\delta^{*} .
\end{aligned}
$$

Thus $q^{\prime} \in \mathscr{M}$ and $\rho\left(q^{\prime}, q\right)<\delta^{*}$.

Since $\left(F\left(q^{\prime}\right) \cap W^{1}\right) \cup\left(F\left(q^{\prime}\right) \cap W^{2}\right)=F\left(q^{\prime}\right) \cap\left(W^{1} \cup W^{2}\right) \neq$ $\emptyset$ and $W^{1} \cap W^{2}=\emptyset$, then we assume $F\left(q^{\prime}\right) \cap W^{1} \neq \emptyset$ without loss of generality. Then there exists $\bar{x} \in F\left(q^{\prime}\right) \cap W^{1}$ such that, for each $i \in I, \bar{x} \in W^{1}, \bar{x}_{i} \in S_{i}^{\prime}(\bar{x})$, and $R_{i}^{\prime}\left(\bar{x}, y_{i}\right)$ holds for any $y_{i} \in Q_{i}^{\prime}(\bar{x})$. When $\bar{x} \in W^{1}$, we have that, for each $i \in I$,

$$
\begin{gathered}
S_{i}^{\prime}(\bar{x})=\lambda(\bar{x}) S_{i}^{1}(\bar{x})+\mu(\bar{x}) S_{i}^{2}(\bar{x})=S_{i}^{1}(\bar{x}), \\
\bar{x} \in B_{i}^{\prime}\left(y_{i}\right)=\left(B_{i}^{1}\left(y_{i}\right) \backslash W^{2}\right) \cup\left(B_{i}^{2}\left(y_{i}\right) \backslash W^{1}\right) \\
\Longrightarrow \bar{x} \in B_{i}^{1}\left(y_{i}\right), \\
Q_{i}^{\prime}(\bar{x})=Q_{i}^{1}(\bar{x}) ;
\end{gathered}
$$

that is, for each $i \in I, \bar{x}_{i} \in S_{i}^{1}(\bar{x})$ and $R_{i}^{1}\left(\bar{x}, y_{i}\right)$ holds for any $y_{i} \in Q_{i}^{1}(\bar{x})$, which implies that $\bar{x} \in F\left(q^{1}\right)$. Hence $F\left(q^{1}\right) \cap$ $W^{1} \neq \emptyset$, which contradicts $F\left(q^{1}\right) \cap W^{1}=\emptyset$. Thus $m(q)$ is connected.

Theorem 13. For each $q \in \mathscr{M}$, there exists at least one essential component of $F(q)$.

Proof. By Theorem 12, there exists at least one connected minimal essential subset $m(q)$ of $F(q)$. Thus, there is a component $C$ of $F(q)$ such that $m(q) \subset C$. It is obvious that $C$ is essential by Remark 2(2). Thus $C$ is an essential component.

Remark 14. If $I$ is a singleton, and $S_{i}(x)=Q_{i}(x)=X_{i}$ for any $x \in X$ and any $i \in I$, the system of quasivariational relations coincides with the variational relation problem considered in [3]. Thus, the results of [3] are obtained as a special case of this paper's results. There, $\mathscr{M}^{\prime}$ is the set of variational relations $R(x, y)$ linking $x, y \in X$ such that (i) $X$ is nonempty convex compact subset of a normed linear space; (ii) for any finite set $\left\{x_{1}, \ldots, x_{n}\right\} \subset X$, and any $x \in \operatorname{co}\left\{x_{1}, \ldots, x_{n}\right\}$, there is $j \in$ $\{1, \ldots, n\}$ such that $R\left(x, x_{j}\right)$ holds; (iii) $R(\cdot, y)$ is closed for any $y \in X$. Then we have the following results, which are Lemma 3.2, Theorems 3.1-3.4 in [3].

Theorem 15. The mapping $F: \mathscr{M}^{\prime} \rightrightarrows X$ is upper semicontinuous with compact values.

Theorem 16. There exists a dense residual subset $\mathscr{G}^{\prime}$ of $\mathscr{M}^{\prime}$ such that, for each $q \in \mathscr{G}^{\prime}, q$ is essential.
Theorem 17. For each $q \in \mathscr{M}^{\prime}$, there exists at least one minimal essential subset of $F(q)$.

Theorem 18. For each $q \in \mathscr{M}^{\prime}$, there exists at least one minimal essential subset of $F(q)$.

Theorem 19. For each $q \in \mathscr{M}^{\prime}$, there exists at least one essential component of $F(q)$.

\section{Applications (I): Systems of KKM Problems}

Let $I$ be any index set. For each $i \in I$, let $X_{i}$ be a nonempty convex compact subset of topological vector space $E_{i}$ and $X=\prod_{i \in I} X_{i}$, and let $S_{i}, Q_{i}: X \rightrightarrows X_{i}$ and $G_{i}: X_{i} \rightrightarrows X$ be multivalued mappings with nonempty values. A system of KKM problems consists in finding $x^{*}=\left(x_{i}^{*}\right)_{i \in I} \in X$ such that, for each $i \in I, x_{i}^{*} \in S_{i}\left(x^{*}\right)$ and $x^{*} \in \bigcap_{y_{i} \in Q_{i}\left(x^{*}\right)} G_{i}\left(y_{i}\right)$.

Definition 20. Let $I$ be a finite set, and $z^{0} \in X$. Let $\mathscr{M}_{1}$ be the set of systems of KKM problems $\eta=\left(I, X_{i}, S_{i}, Q_{i}, G_{i}\right)$ such that, for each $i \in I$, (1) $X_{i}$ is a nonempty convex compact subset of a normed linear space $E_{i}$; (2) $z_{i}^{0} \in Q_{i}(x) \subset S_{i}(x)$ for any $x \in X$ and any $i \in I$, and $Q_{i}^{-1}\left(y_{i}\right)$ is open in $X$ for all $y_{i} \in X_{i}$; (3) $S_{i}$ is upper semicontinuous with nonempty compact convex values; (4) the multivalued mapping $G_{i}$ : $X_{i} \rightrightarrows X$ holds nonempty closed values; (5) for any finite set $\left\{x_{i 1}, \ldots, x_{i n}\right\} \subset X_{i}$, and any $x_{i} \in \operatorname{co}\left\{x_{i 1}, \ldots, x_{i n}\right\}$, there is $j \in\{1, \ldots, n\}$ such that $x \in G_{i}\left(x_{i j}\right)$ holds, where $x=\left(x_{i}\right)_{i \in I}$.

For each $\eta \in \mathscr{M}_{1}$, denote by $F_{1}(\eta)$ the solution set of $\eta$ and $q^{\eta} \in \mathscr{M}$ by $q^{\eta}=\left(S_{i}, Q_{i}, R_{i}^{\eta}\right)_{i \in I}$, where

$$
R_{i}^{\eta}\left(x, y_{i}\right) \text { holds if and only if } x \in G_{i}\left(y_{i}\right), \quad \forall i \in I .
$$

Easily $F_{1}(\eta)$ is nonempty. Furthermore, for any $\eta, \eta^{\prime} \in \mathscr{M}_{1}$, we define the distance on $\mathscr{M}_{1}$ by $\rho_{1}\left(\eta, \eta^{\prime}\right)=\rho\left(q^{\eta}, q^{\eta^{\prime}}\right)$. Thus there exists an isometric mapping $T_{1}: \mathscr{M}_{1} \rightarrow \mathscr{M}$ such that $T_{1}(\eta)=q^{\eta}$ and $F_{1}(\eta)=F\left(q^{\eta}\right)=F\left(T_{1}(\eta)\right)$.

Theorem 21. For each $\eta \in \mathscr{M}_{1}$, there exists at least one essential component of $F_{1}(\eta)$.

Proof. Since $T_{1}: \mathscr{M}_{1} \rightarrow \mathscr{M}$ is an isometric mapping such that $T_{1}(\eta)=q^{\eta}$, it is continuous. By Theorem 13, there exists at least one essential component of $F(q)$ for each $q \in \mathscr{M}$, and by Lemma 7, there exists at least one essential component of $F_{1}(\eta)$ for each $\eta \in \mathscr{M}_{1}$.

\section{Applications (II): Systems of Variational Inclusions}

Let $I$ be any index set. For each $i \in I$, let $X_{i}$ be a nonempty subset of Hausdorff topological space, let $Z_{i}$ be a Hausdorff topological vector space, and let $S_{i}, Q_{i}: X \rightrightarrows X_{i}, A_{i}, B_{i}: X \times$ $X_{i} \rightrightarrows Z_{i}$ be multivalued mappings with nonempty values. 
A system of quasivariational inclusions of type (I) consists in finding $x^{*} \in X$ such that, for each $i \in I, x_{i}^{*} \in S_{i}\left(x^{*}\right)$ and $A_{i}\left(x^{*}, y_{i}\right) \subset B_{i}\left(x^{*}, y_{i}\right)$ for all $y_{i} \in Q_{i}\left(x^{*}\right)$.

A system of quasivariational inclusions of type (II) consists in finding $x^{*} \in X$ such that, for each $i \in I, x_{i}^{*} \in S_{i}\left(x^{*}\right)$ and $A_{i}\left(x^{*}, y_{i}\right) \cap B_{i}\left(x^{*}, y_{i}\right) \neq \emptyset$ for all $y_{i} \in Q_{i}\left(x^{*}\right)$.

A system of quasivariational inclusions of type (III) consists in finding $x^{*} \in X$ such that, for each $i \in I, x_{i}^{*} \in$ $S_{i}\left(x^{*}\right)$ and $0 \in A_{i}\left(x^{*}, y_{i}\right)$ for all $y_{i} \in Q_{i}\left(x^{*}\right)$.

Definition 22. Let $I$ be a finite set, and $z^{0} \in X$. Let $\mathscr{M}_{2}^{1}$ be the set of systems of quasivariational inclusions of type (I) $\eta=\left(I, X_{i}, S_{i}, Q_{i}, A_{i}, B_{i}\right)$ such that, for each $i \in I$, (1) $X_{i}$ is a nonempty convex compact subset of a normed linear space $E_{i}$; (2) $z_{i}^{0} \in Q_{i}(x) \subset S_{i}(x)$ for any $x \in X$ and any $i \in I$, and $Q_{i}^{-1}\left(y_{i}\right)$ is open in $X$ for all $y_{i} \in X_{i}$; (3) $S_{i}$ is upper semicontinuous with nonempty compact convex values; (4) for any $y_{i} \in X_{i}, A_{i}\left(\cdot, y_{i}\right)$ is lower semicontinuous, and $B_{i}\left(\cdot, y_{i}\right)$ is closed; (5) for any $\left\{x_{i 1}, \ldots, x_{i n}\right\}$ of $X_{i}$ and any $x_{i} \in \operatorname{co}\left\{x_{i 1}, \ldots, x_{i n}\right\}$, there is $j \in\{1, \ldots, n\}$ such that $A_{i}\left(x, x_{i j}\right) \subset B_{i}\left(x, x_{i j}\right)$, where $x=\left(x_{i}\right)_{i \in I}$.

For each $\eta \in \mathscr{M}_{2}^{1}$, denote by $F_{2}^{1}(\eta)$ the solution set of $\eta$. For any $\eta \in \mathscr{M}_{2}^{1}$, define $q^{\eta}$ by $q^{\eta}=\left(S_{i}, Q_{i}, R_{i}^{\eta}\right)_{i \in I}$, where

$$
R_{i}^{\eta}\left(x, y_{i}\right) \text { holds if and only if } A_{i}\left(x, y_{i}\right) \subset B_{i}\left(x, y_{i}\right) \text {, }
$$

$\forall i \in I$.

By Theorem 3.5 of [8], $q^{\eta} \in \mathscr{M}$. Easily, $F_{2}^{1}(\eta)$ is nonempty. Further, for any $\eta, \eta^{\prime} \in \mathscr{M}_{2}^{1}$, we define the distance on $\mathscr{M}_{2}^{1}$ by $\rho_{2}^{1}\left(\eta, \eta^{\prime}\right)=\rho\left(q^{\eta}, q^{\eta^{\prime}}\right)$. Thus there exists an isometric mapping $T_{2}^{1}: \mathscr{M}_{2}^{1} \rightarrow \mathscr{M}$ such that $T_{2}^{1}(\eta)=q^{\eta}$ and $F_{2}^{1}(\eta)=F\left(q^{\eta}\right)=$ $F\left(T_{2}^{1}(\eta)\right)$.

Theorem 23. For each $\eta \in \mathscr{M}_{2}^{1}$, there exists at least one essential component of $F_{2}^{1}(\eta)$.

Proof. Since $T_{2}^{1}: \mathscr{M}_{2}^{1} \rightarrow \mathscr{M}$ is an isometric mapping such that $T_{2}^{1}(\eta)=q^{\eta}$, it is continuous. By Theorem 13, there exists at least one essential component of $F(q)$ for each $q \in \mathscr{M}$, and by Lemma 7 , there exists at least one essential component of $F_{2}^{1}(\eta)$ for each $\eta \in \mathscr{M}_{2}^{1}$.

It is similar to Definition 22 and Theorem 23, and the following results are obtained. Here, we do not repeat the process.

Definition 24. Let $I$ be a finite set, and $z^{0} \in X$. Let $\mathscr{M}_{2}^{2}$ be the set of systems of quasivariational inclusions of type (II) $\eta=\left(I, X_{i}, S_{i}, Q_{i}, A_{i}, B_{i}\right)$ such that, for each $i \in I,(1) X_{i}$ is a nonempty convex compact subset of a normed linear space $E_{i}$; (2) $z_{i}^{0} \in Q_{i}(x) \subset S_{i}(x)$ for any $x \in X$ and any $i \in I$, and $Q_{i}^{-1}\left(y_{i}\right)$ is open in $X$ for all $y_{i} \in X_{i}$; (3) $S_{i}$ is upper semicontinuous with nonempty compact convex values; (4) for any $y_{i} \in X_{i}, A_{i}\left(\cdot, y_{i}\right)$ is upper semicontinuous with nonempty compact values and $B_{i}\left(\cdot, y_{i}\right)$ is closed; (5) for any $\left\{x_{i 1}, \ldots, x_{i n}\right\}$ of $X_{i}$ and any $x_{i} \in \operatorname{co}\left\{x_{i 1}, \ldots, x_{i n}\right\}$, there is $j \epsilon$ $\{1, \ldots, n\}$ such that $A_{i}\left(x, x_{i j}\right) \cap B_{i}\left(x, x_{i j}\right) \neq \emptyset$, where $x=$ $\left(x_{i}\right)_{i \in I}$.

Definition 25. Let $I$ be a finite set, and $z^{0} \in X$. Let $\mathscr{M}_{2}^{3}$ be the set of systems of quasivariational inclusions of type (III) $\eta=$ $\left(I, X_{i}, S_{i}, Q_{i}, A_{i}\right)$ such that, for each $i \in I,(1) X_{i}$ is a nonempty convex compact subset of a normed linear space $E_{i} ;(2) z_{i}^{0} \epsilon$ $Q_{i}(x) \subset S_{i}(x)$ for any $x \in X$ and any $i \in I$, and $Q_{i}^{-1}\left(y_{i}\right)$ is open in $X$ for all $y_{i} \in X_{i}$; (3) $S_{i}$ is upper semicontinuous with nonempty compact convex values; (4) for any $y_{i} \in X_{i}$, $A_{i}\left(\cdot, y_{i}\right)$ is closed; (5) for any $\left\{x_{i 1}, \ldots, x_{i n}\right\}$ of $X_{i}$ and any $x_{i} \in$ $\operatorname{co}\left\{x_{i 1}, \ldots, x_{i n}\right\}$, there is $j \in\{1, \ldots, n\}$ such that $\mathbf{0} \in A_{i}\left(x, x_{i j}\right)$, where $x=\left(x_{i}\right)_{i \in I}$.

For each $j=2,3$ and each $\eta \in \mathscr{M}_{2}^{j}$, denote the solution set of $\eta$ by $F_{2}^{j}(\eta)$.

Theorem 26. For $j=2,3$ and each $\eta \in \mathscr{M}_{2}^{j}$, there exists at least one essential component of $F_{2}^{j}(\eta)$.

\section{Conclusions}

In this paper, we study the notions of essential stability of solutions for system of quasivariational relations. We show that most of systems of quasivariational relations (in the sense of Baire category) are essential and that, for any system of quasivariational relations, there exists at least one essential component of its solution set. As applications, the existence of essential components of solution set for systems of KKM problems and systems of quasivariational inclusions is obtained.

\section{Conflict of Interests}

The authors declare that there is no conflict of interests regarding the publication of this paper.

\section{Authors' Contribution}

All authors contributed equally and significantly in writing this paper. All authors read and approved the final paper.

\section{Acknowledgments}

This research was supported by project supported by the China's Post-Doctoral Science Fund (no. 2014M552325), the Chen Guang Project sponsored by the Shanghai Municipal Education Commission and Shanghai Education Development Foundation (no. 13CG35), the open project of Key Laboratory of Mathematical Economics (SUFE), Ministry of Education (no. 201309KF02), and the Chongqing Foundation and Cutting-Edge Research Program (no. cstc2014jcyjA90016). 


\section{References}

[1] D. T. Luc, "An abstract problem in variational analysis," Journal of Optimization Theory and Applications, vol. 138, no. 1, pp. 6576, 2008.

[2] P. Q. Khanh and D. T. Luc, "Stability of solutions in parametric variational relation problems," Set-Valued Analysis, vol. 16, no. 7-8, pp. 1015-1035, 2008.

[3] Y. J. Pu and Z. Yang, "Stability of solutions for variational relation problems with applications," Nonlinear Analysis: Theory, Methods \& Applications, vol. 75, no. 4, pp. 1758-1767, 2012.

[4] L. Lin and S. Wang, "Simultaneous variational relation problems and related applications," Computers \& Mathematics with Applications, vol. 58, no. 9, pp. 1711-1721, 2009.

[5] M. Balaj and D. T. Luc, "On mixed variational relation problems," Computers \& Mathematics with Applications, vol. 60, no. 9, pp. 2712-2722, 2010.

[6] M. Balaj and L. Lin, "Equivalent forms of a generalized KKM theorem and their applications," Nonlinear Analysis: Theory, Methods \& Applications, vol. 73, no. 3, pp. 673-682, 2010.

[7] M. Balaj and L. J. Lin, "Generalized variational relation problems with applications," Journal of Optimization Theory and Applications, vol. 148, no. 1, pp. 1-13, 2011.

[8] L. Lin and Q. H. Ansari, "Systems of quasi-variational relations with applications," Nonlinear Analysis: Theory, Methods \& Applications, vol. 72, no. 3-4, pp. 1210-1220, 2010.

[9] D. T. Luc, E. Sarabi, and A. Soubeyran, "Existence of solutions in variational relation problems without convexity," Journal of Mathematical Analysis and Applications, vol. 364, no. 2, pp. 544555, 2010.

[10] Y. J. Pu and Z. Yang, "Variational relation problem without the KKM property with applications," Journal of Mathematical Analysis and Applications, vol. 393, no. 1, pp. 256-264, 2012.

[11] Z. Yang and Y. J. Pu, "Generalized Knaster-KuratowskiMazurkiewicz theorem without convex hull," Journal of Optimization Theory and Applications, vol. 154, no. 1, pp. 17-29, 2012.

[12] Z. Yang and Y. J. Pu, "Existence and stability of solutions for maximal element theorem on Hadamard manifolds with applications," Nonlinear Analysis: Theory, Methods \& Applications, vol. 75, no. 2, pp. 516-525, 2012.

[13] R. P. Agarwal, M. Balaj, and D. O'Regan, "A unifying approach to variational relation problems," Journal of Optimization Theory and Applications, vol. 155, no. 2, pp. 417-429, 2012.

[14] M. Balaj and L. J. Lin, "Existence criteria for the solutions of two types of variational relation problems," Journal of Optimization Theory and Applications, vol. 156, no. 2, pp. 232-246, 2013.

[15] E. van Damme, Stability and Perfection of Nash Equilibria, Springer, Berlin, Germany, 1987.

[16] M. K. Fort Jr., "Essential and non essential fixed points," The American Journal of Mathematics, vol. 72, pp. 315-322, 1950.

[17] W. T. Wu and J. H. Jiang, "Essential equilibrium points of nperson noncooperative games," Scientia Sinica, vol. 11, pp. 13071322, 1962.

[18] J. H. Jiang, "Essential component of the set of fixed points of the multivalued mappings and its application to the theory of games," Scientia Sinica, vol. 12, pp. 951-964, 1963.

[19] S. Kinoshita, "On essential components of the set of fixed points," vol. 4, pp. 19-22, 1952.

[20] E. Kohlberg and J. F. Mertens, "On the strategic stability of equilibria,” Econometrica, vol. 54, no. 5, pp. 1003-1037, 1986.
[21] J. Yu and S. Xiang, "On essential components of the set of Nash equilibrium points," Nonlinear Analysis: Theory, Methods \& Applications, vol. 38, pp. 259-264, 1999.

[22] J. Yu and Q. Luo, "On essential components of the solution set of generalized games," Journal of Mathematical Analysis and Applications, vol. 230, no. 2, pp. 303-310, 1999.

[23] H. Yang and J. Yu, "Essential components of the set of weakly Pareto-Nash equilibrium points," Applied Mathematics Letters, vol. 15, no. 5, pp. 553-560, 2002.

[24] J. Yu and Y. Zhou, "A Hausdorff metric inequality with applications to the existence of essential components," Nonlinear Analysis: Theory, Methods \& Applications, vol. 69, no. 5-6, pp. 1851-1855, 2008.

[25] J. Yu, H. Yang, and S. Xiang, "Unified approach to existence and stability of essential components," Nonlinear Analysis, Theory, Methods and Applications, vol. 63, no. 5-7, pp. e2415-e2425, 2005.

[26] Z. Yang and Y. J. Pu, "Essential stability of solutions for maximal element theorem with applications," Journal of Optimization Theory and Applications, vol. 150, no. 2, pp. 284-297, 2011.

[27] Z. Yang and Y. J. Pu, "Existence and stability of minimax regret equilibria," Journal of Global Optimization, vol. 54, no. 1, pp. 1726, 2012.

[28] Z. Yang and Y. J. Pu, "On existence and essential components for solution set for system of strong vector quasi-equilibrium problems," Journal of Global Optimization, vol. 55, no. 2, pp. 253-259, 2013.

[29] Y. H. Zhou, J. Yu, and S. W. Xiang, "Essential stability in games with infinitely many pure strategies," International Journal of Game Theory, vol. 35, no. 4, pp. 493-503, 2007.

[30] P. Q. Khanh and N. H. Quan, "Generic stability and essential components of generalized KKM points and applications," Journal of Optimization Theory and Applications, vol. 148, no. 3, pp. 488-504, 2011.

[31] M. K. Fort Jr., "A unified theory of semi-continuity," Duke Mathematical Journal, vol. 16, no. 2, pp. 237-246, 1949.

[32] J. P. Aubin and I. Ekeland, Applied Nonlinear Analysis, John Wiley \& Sons, New York, NY, USA, 1984. 


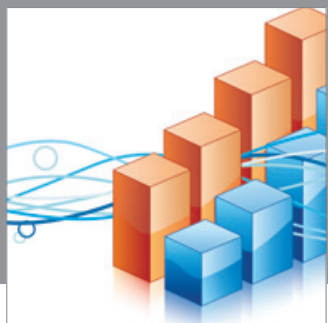

Advances in

Operations Research

mansans

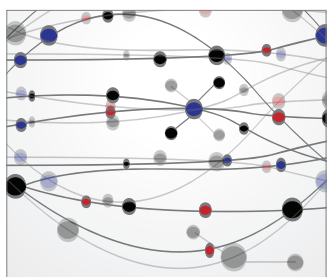

The Scientific World Journal
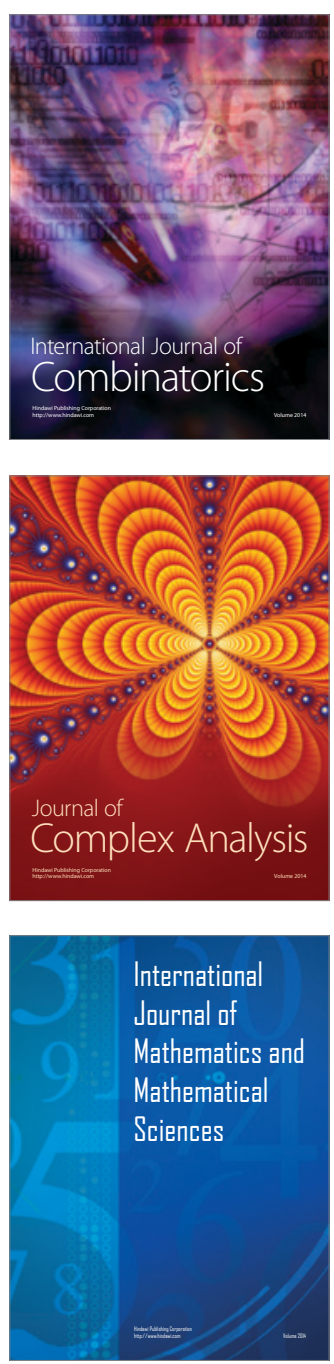
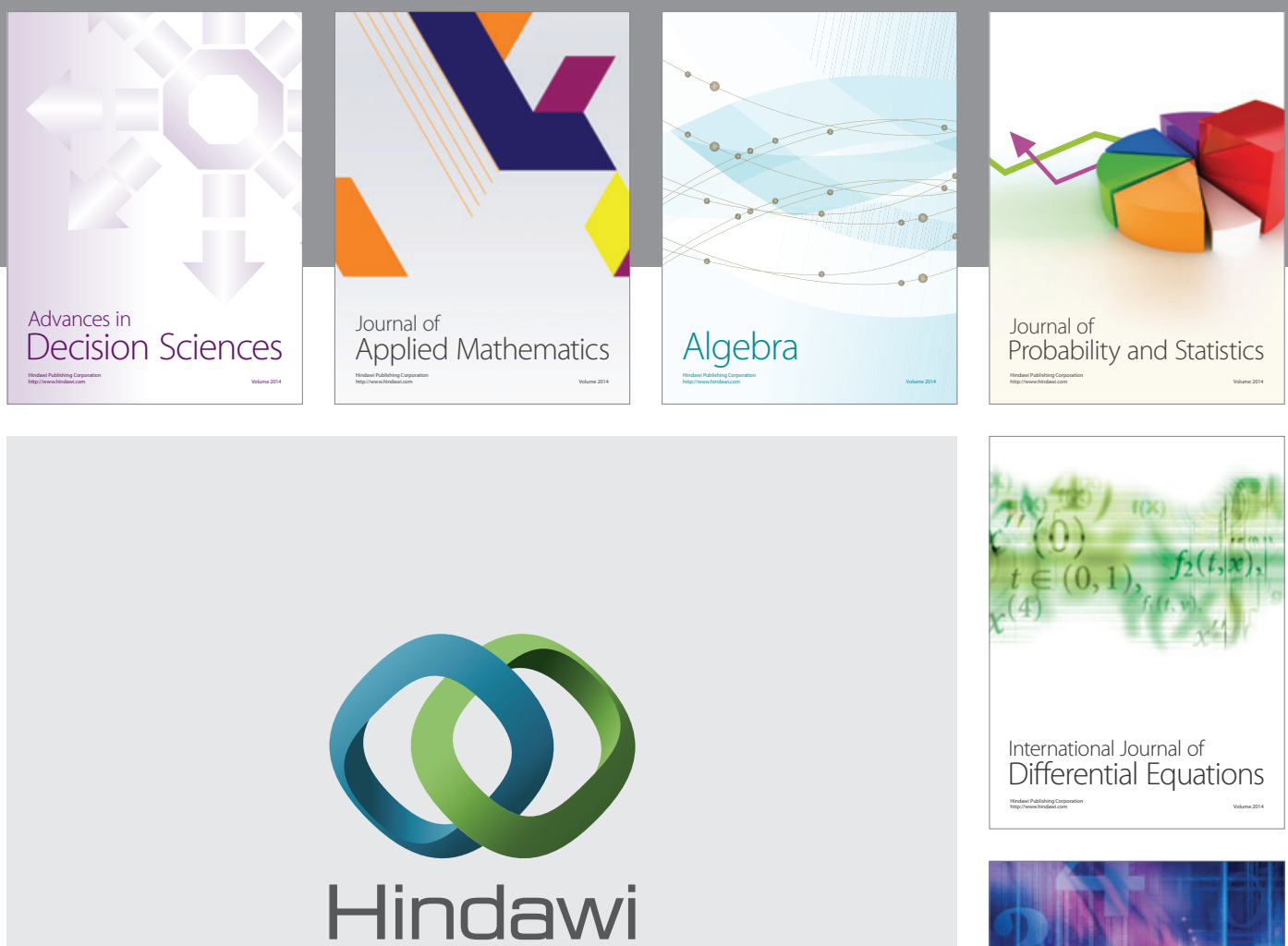

Submit your manuscripts at http://www.hindawi.com
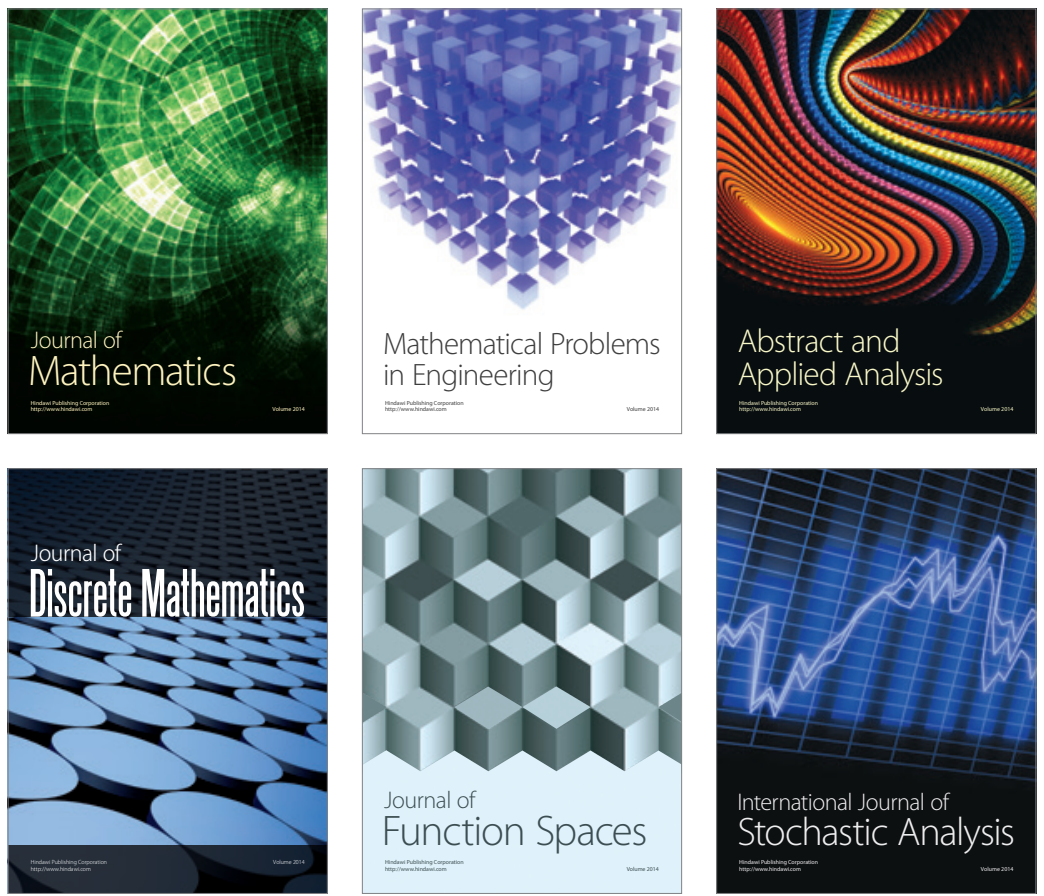

Journal of

Function Spaces

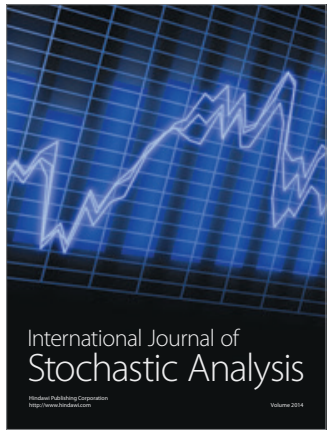

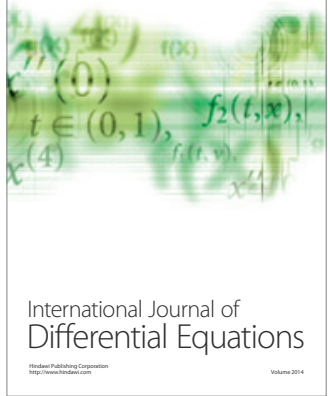
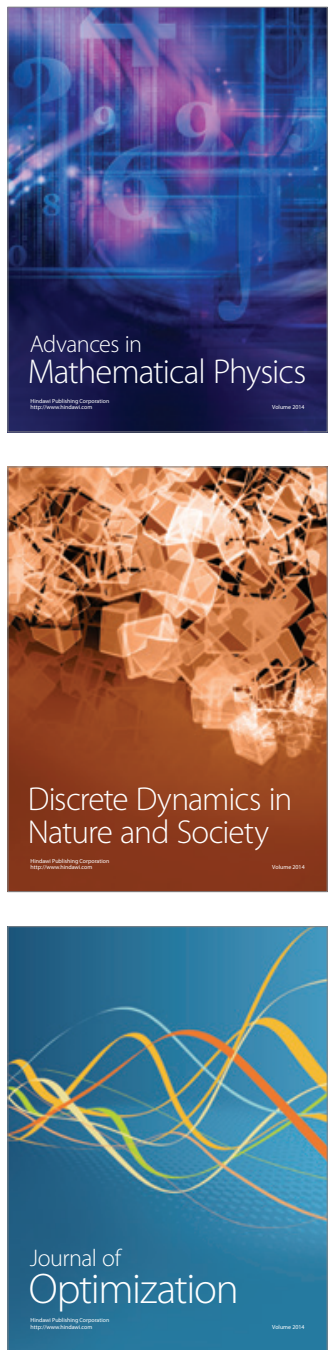